\title{
Synchronization of Optomechanical Nanobeams by Mechanical Interaction
}

\author{
M. F. Colombano, ${ }^{1,2, *}$ G. Arregui, ${ }^{1,2,{ }^{*}}$ N.E. Capuj,${ }^{3,4}$ A. Pitanti, ${ }^{5}$ J. Maire, ${ }^{1}$ A. Griol, ${ }^{6}$ B. Garrido, ${ }^{7}$ \\ A. Martinez, ${ }^{6}$ C. M. Sotomayor-Torres, ${ }^{1,8}$ and D. Navarro-Urrios ${ }^{7, \dagger}$ \\ ${ }^{1}$ Catalan Institute of Nanoscience and Nanotechnology (ICN2), CSIC and The Barcelona Institute of Science and Technology, \\ Campus UAB, Bellaterra, 08193 Barcelona, Spain \\ ${ }^{2}$ Dept. de Fìsica, Universitat Autònoma de Barcelona, 08193 Bellaterra, Spain \\ ${ }^{3}$ Depto. Física, Universidad de La Laguna, 38200 San Cristóbal de La Laguna, Spain \\ ${ }^{4}$ Instituto Universitario de Materiales y Nanotecnología, Universidad de La Laguna, 38071 Santa Cruz de Tenerife, Spain \\ ${ }^{5}$ NEST, CNR_Istituto Nanoscienze and Scuola Normale Superiore, Piazza San Silvestro 12, 56127 Pisa, Italy \\ ${ }^{6}$ Nanophotonics Technology Center, Universitat Politècnica de Valencia, 46022 Valencia, Spain \\ ${ }^{7}$ MIND-IN2UB, Departament d'Enginyerìa Electrònica i Biomèdica, Facultat de Fìsica, Universitat de Barcelona, \\ Martì i Franquès 1, 08028 Barcelona, Spain \\ ${ }^{8}$ ICREA_Instituciò Catalana de Recerca i Estudis Avançats, 08010 Barcelona, Spain
}

(Received 16 October 2018; published 1 July 2019)

\begin{abstract}
The synchronization of coupled oscillators is a phenomenon found throughout nature. Mechanical oscillators are paradigmatic examples, but synchronizing their nanoscaled versions is challenging. We report synchronization of the mechanical dynamics of a pair of optomechanical crystal cavities that, in contrast to previous works performed in similar objects, are intercoupled with a mechanical link and support independent optical modes. In this regime they oscillate in antiphase, which is in agreement with the predictions of our numerical model that considers reactive coupling. We also show how to temporarily disable synchronization of the coupled system by actuating one of the cavities with a heating laser, so that both cavities oscillate independently. Our results can be upscaled to more than two cavities and pave the way towards realizing integrated networks of synchronized mechanical oscillators.
\end{abstract}

DOI: 10.1103/PhysRevLett.123.017402

Synchronization of autonomous oscillators, first observed back in the 17th century by Lord Huygens [1], manifests itself throughout nature extending from subatomic to cosmic scales, covering widely different research topics from biology to astrophysics [2-5]. This explains the existence of the vast literature devoted to synchronized oscillator networks, which have attracted much interest for decades [6-8], in part due to their applicability in neural networks [9]. With the advent of nanotechnologies, large efforts have been dedicated to synchronizing oscillating nanoelectromechanical systems (NEMS), which have wide practical applications because of their scalable architecture and accurate control of operating frequencies and quality factors by design [10-12]. Self-driven synchronized NEMS oscillator networks will find a variety of additional applications, such as on-chip robust time keeping [13] and mass [14], gas [15,16], and force sensors [17] with extremely low phase noise [2]. The impressive progress in cavity optomechanics during the last decade [18-22] has evidenced that optomechanical (OM) oscillators are also ideal building blocks for the observation, control, and exploitation of synchronization phenomena [23]. However, the field of OM oscillator networks is still in its infancy, being restricted to purely theoretical work and experiment proposals $[23,24]$.
Essential conditions for spontaneous synchronization between two dynamical systems are that [2] (i) both of them are self-sustained oscillators, i.e., capable of generating their own rhythms, (ii) the systems adjust their rhythm due to a weak interaction, and (iii) the adjustment of rhythms occurs in a certain range of mismatch of the individual systems. To date, there has been only a handful of reports claiming synchronization in OM cavities sharing a common optical mode that drive both cavities [25-27] and another in which frequency locking in optically coupled cascaded resonators was achieved [28]. On the other hand, a couple of works have reported long-range synchronization between $\mathrm{OM}$ cavities placed in different chips [29-31]. The coupling mechanism in these latter cases relies on modulating the optical excitation of one of the cavities with an electro-optical modulator that reproduces the dynamics of the other cavity. Concerning NEMS with purely mechanical coupling, Shim et al. [10] reported a resonant excitation of coupled resonators using an external source; i.e., the oscillators were not self-sustained. In this Letter, we unambiguously demonstrate spontaneous synchronization of the coherent mechanical motion of a pair of one-dimensional silicon OM photonic crystals (OMCs) integrated in the same chip. The OMCs interact weakly means of an engineered mechanical link, thus 

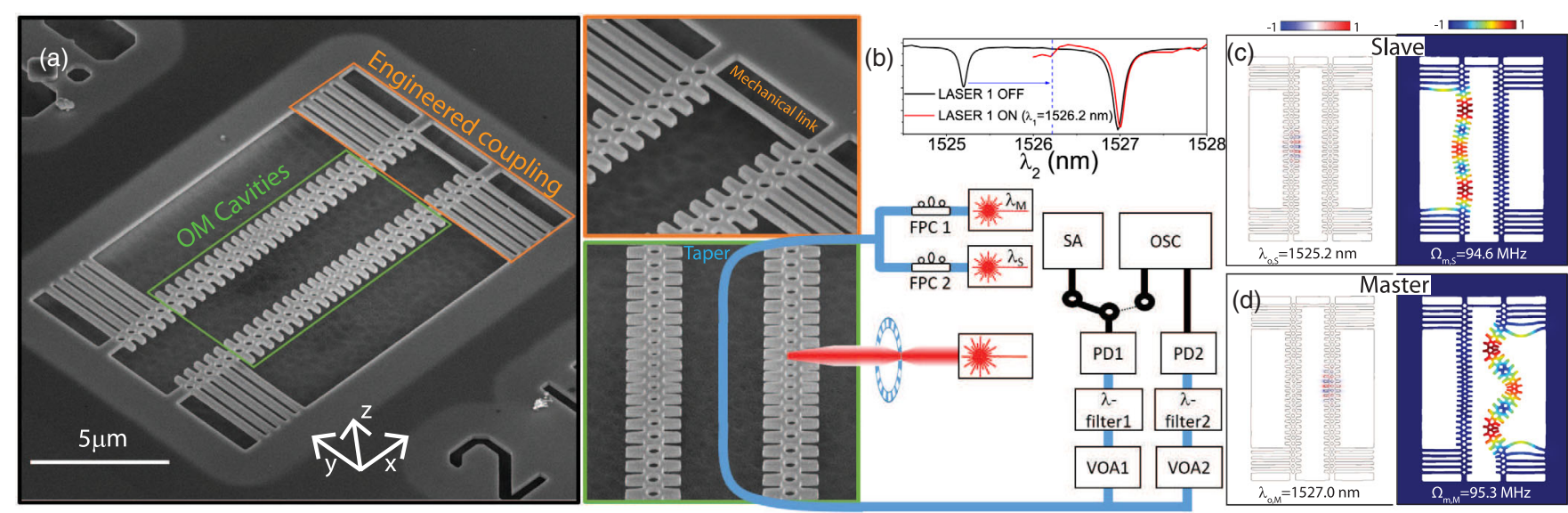

FIG. 1. Mechanically coupled optomechanical crystal cavities. (a) SEM image of the pair of OMCs under study. (b) Schematic of the experimental setup. The optical transmission of a single cavity can be isolated from the other by means of $\lambda$ filters. Variable optical attenuator (VOA); fiber polarizer controller (FPC); spectrum analyzer (SA); oscilloscope (OSC); photodetector (PD). The top subpanel shows a transmission spectrum displaying resonances belonging to each cavity (black) and a spectrum of the longer-wavelength resonance while redshifting the first (red). (c) and (d) FEM simulations of the normalized optical Ey field and the mechanical displacement field $\|Q\|$ of the optical and mechanical modes under study using an imported geometry measured by SEM. The eigenmodes associated with the left (slave) and right (master) OMCs are depicted in panels (c) and (d), respectively. The displacement profiles have been altered to illustrate the larger oscillation amplitude of the master.

avoiding the need for external feedback loop schemes. In contrast to the previous works in literature [25-28], the OMCs are optically isolated from each other, are independently measured, and individually driven to a state of high-amplitude, coherent, and self-sustained mechanical motion (mechanical lasing from now on) using the anharmonic modulation of the radiation pressure force due to the activation of a self-pulsing (SP) regime described in detail in the Supplemental Material [32] and elsewhere [33-35]. Essentially, the SP limit cycle is the solution of the system of coupled differential equations governing the free-carrier concentration and the effective temperature of the cavity region within a wide range of excitation laser wavelengths and powers. That dynamical interplay between free-carrierdispersion (FCD) and the thermo-optic (TO) effect implies an oscillation of the cavity resonance around the laser line at a frequency $\left(\nu_{\mathrm{SP}}\right)$ that depends on the laser parameters. If a mechanical mode is partially resonant with one of the harmonics of $\nu_{\mathrm{SP}}$, it can be driven into mechanical lasing. Moreover, by means of the OM coupling, the mechanical motion frequency entrains the SP such that $\nu_{\mathrm{SP}}$ is forced to be a simple fraction of the mechanical eigenfrequency. An important feature of this regime is that its frequency can be thermally tuned by modifying the laser wavelength, given that the average temperature of the OMC increases with the laser wavelength around which the mechanical lasing limit cycle takes place due to a larger average intracavity photon number $\left(n_{o}\right)$.

The device investigated here is a pair of nominally equivalent one-dimensional OMCs fabricated using standard Si nanofabrication processes (see Supplemental Material [32]) in a silicon-on-insulator wafer [Fig. 1(a)].
The five outer cells at each side of the crystals are clamped to the partially underetched Si frame, so that the in-plane flexural modes are decoupled from the frame and confined to the central region of the OMCs, which are designed to support high-Q optical cavity modes at around $1.53 \mu \mathrm{m}$. The OMCs are mechanically interconnected on one side by a tether linking the inner stubs of the last cells. The physical separation between the crystals $(\sim 2 \mu \mathrm{m})$ is large enough to prevent optical crosstalk while allowing for their simultaneous optical excitation using a single tapered fiber that is placed in between.

In order to check whether the observed optical resonances belong to different OMCs we excite one of the resonances and redshift it using the TO effect while simultaneously monitoring the spectral position of the other resonance with the second laser [see the experimental setup in Fig. 1(b)]. If only one resonance shifts it means that the two resonances belong to different OMCs, as in the top panel of Fig. 1(b).

We investigate the fundamental optical cavity mode [Fig. 1(c)] of each OMC. These modes display high values of single-particle OM coupling rate $\left(g_{o}\right)$ for in-plane (xy plane) flexural modes. In particular, the ones having three antinodes along the $x$ direction [Fig. 1(d)] display a frequency of $\Omega_{M}=95.3$ and $\Omega_{S}=94.6 \mathrm{MHz}$ in each OMC and a calculated value of $g_{o, M} / 2 \pi=514$ and $g_{o, S} / 2 \pi=330 \mathrm{kHz}$, respectively (see Supplemental Material [32], which includes Refs. [36-38]). Hereinafter, we adopt the notation $M$ and $S$ to denote master and slave, where the OMC displaying higher $\Omega$ and longer optical resonance wavelengths is the master and the other the slave. It is worth remarking that both oscillators can freely adapt 


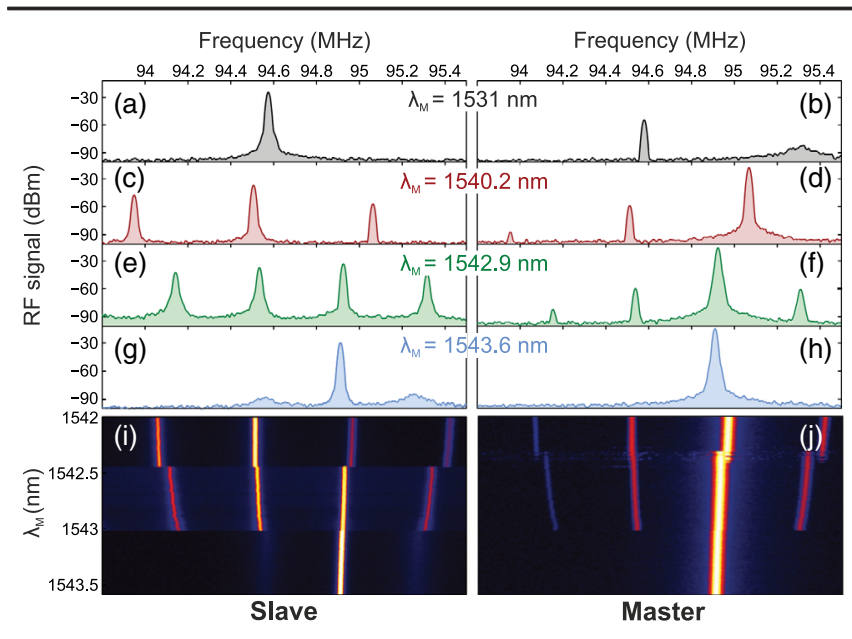

FIG. 2. Synchronization of optomechanical oscillators in the frequency domain. (a)-(h) Radio-frequency spectra of the optical transmission associated to the slave (left panels) and the master (right panels) for different values of the wavelength of the laser driving the master $\left(\lambda_{M}\right)$. The wavelength of the laser driving the slave is fixed at $\lambda_{S}=1529 \mathrm{~nm}$. (i)-(j) Color contour plots of the rf spectra as a function of $\lambda_{M}$ of the slave and master [(i) and (j), respectively].

their oscillation phase and that the notation master or slave must not be associated with any sort of injection locking scheme. It reflects the fact that, in mechanical lasing, the master oscillation amplitude is significantly larger, so that its dynamics is much less sensible to the slave oscillations than the other way around.

The experiment that demonstrates synchronization of the dynamics of the two OMCs is illustrated in Fig. 2, where the left and right panels report the radio-frequency (rf) spectra of the optical transmission associated to the laser exciting the slave and master, respectively. One laser is first switched on in between the two optical resonances and used to push the resonance of the master to $\lambda_{M}=1531 \mathrm{~nm}$ using the TO effect. The second laser is then used to excite the resonance of the slave at $\lambda_{S}=1529 \mathrm{~nm}$, where it sets up a mechanical lasing regime at $94.6 \mathrm{MHz}$. A sharp and intense rf tone is observed when $\lambda$-filterl is tuned at $\lambda_{S}=$ $1529 \mathrm{~nm}$ [Fig. 2(a)]. The incident power values are $P_{i n, M}=$ $8 \mathrm{~mW}$ and $P_{i n, S}=3 \mathrm{~mW}$, respectively. The master is mechanically excited by the coherent motion of the slave as a consequence of the mechanical intercoupling of both OMCs. Indeed, when detecting at $\lambda_{M}=1531 \mathrm{~nm}$ this excitation appears as a sharp rf tone at $94.6 \mathrm{MHz}$ [Fig. 2(b)]. A broad peak centered at $95.3 \mathrm{MHz}$ is also present under this configuration, which is associated to the transduction of the thermally activated motion of the mechanical mode localized in the master. By further redshifting $\lambda_{M}$ up to $\lambda_{M}=1540.2 \mathrm{~nm}$, a mechanical lasing regime is also established in the master, where $\Omega_{M}$ decreases due to material heating. This latter effect appears to be also experienced by $\Omega_{S}$ to a weaker extent; i.e., part of the heat generated in the master could end up increasing the effective temperature of the slave. However, it is also plausible that the master is frequency pulling the slave towards lower frequencies [39]. At this point, since both OMCs are in a mechanical lasing regime, the spectral oscillation amplitude of the optical resonance is much larger than its linewidth and the transduction becomes extremely nonlinear [40]. Therefore, an additional rf peak appears at the beating frequency $\left(\Omega_{M}-\Omega_{S}\right)$ together with symmetric sidebands at both sides of the lasing tones of the OMCs, which still lase at their own rhythm [Figs. 2(c) and 2(d)]. The comparison of the rf spectra of both OMCs shows that the mechanical amplitude of the master is much larger than that of the slave as expected from the larger $g_{o, M}$ and $P_{\mathrm{in}, M}$ values. The dynamical state of the two OMCs remain qualitatively the same until $\lambda_{M}$ reaches $\lambda_{M}=1542.3 \mathrm{~nm}$, where the coupled system enters into a transition region. There, the slave displays a complex rf spectrum of multiple peaks in which the main one is at $\Omega_{M}$ [see Figs. 2(e) and 2(f), where $\lambda_{M}=1542.9 \mathrm{~nm}$ ]. This state is not yet synchronization, even though the slave is strongly affected by the dynamics of the master, the resulting state being of a quite complex nature. Synchronization is achieved above $\lambda_{M}=1543 \mathrm{~nm}$, where both OMCs coherently oscillate at $\Omega_{\text {Sync }} \approx \Omega_{M}$ [see Figs. 2(g) and 2(h), where $\left.\lambda_{M}=1543.6 \mathrm{~nm}\right]$. Both $\mathrm{rf}$ spectra look very similar with the remarkable difference of the presence of broad sidebands on the signal corresponding to the slave, which are absent from the $\mathrm{rf}$ spectra of the master. Those sidebands are clear signatures of master-slave synchronization and have been reported and analyzed in previous works addressing synchronization of photonic cavities [27]. Their origin lies in the effect of the thermal force noise on the dynamics of the system. These forces push the slave phase trajectory out from the limit cycle of the synchronized state, returning back in an oscillatory fashion at a frequency of $\left(\Omega_{M}-\Omega_{S}\right)$. On the contrary, no sidebands appear on the master spectrum, as it overdampedly returns to the limit cycle when driven away from it (see Supplemental Material [32]). The color 2D plots of Figs. 2(i) and 2(j) reveal that the definition of the coupled system as a master-slave one is an oversimplification since $\Omega_{M}$ is also slightly pulled towards $\Omega_{S}$. This is evidenced in the abrupt frequency jumps of $\Omega_{M}$ both when entering the transition region above $\lambda_{M}=1542.3 \mathrm{~nm}$ and the synchronized states above $\lambda_{M}=1543 \mathrm{~nm}$.

The temporal traces of the transmitted optical signal associated with each OMC are simultaneously recorded (Fig. 3) over a time span of $4 \mu$ s. In Fig. 3(a) we report the first $300 \mathrm{~ns}$ and an enlargement of a single period appears in Fig. 3(b). In order to analyze the quality of the synchronization signal, in Fig. 3(c) we show the Poincaré map associated with both temporal traces using a stroboscopic technique; i.e., we collect the pair of values transmission master, transmission slave at a specific sampling frequency. If the oscillators are synchronized and the temporal traces are sampled at $\Omega_{\text {Sync }}$, a point on the Poincaré map is always 

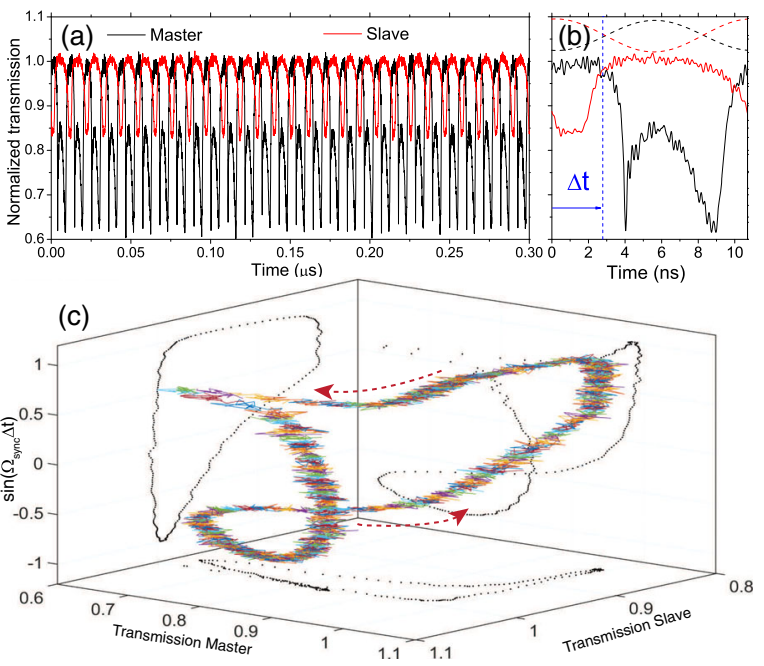

FIG. 3. Temporal traces and Poincaré map of the synchronized state. (a) Temporal traces of the optical transmission of the master and slave cavities (black and red curves, respectively) as recorded simultaneously in the two channels of the oscilloscope. (b) Enlargement of a single period of the two signals. The simulated normalized generalized mechanical deformation corresponding to the experimental transmission traces are also reported (dashed lines). (c) Poincaré map of the full temporal traces using the stroboscopic technique with a sampling frequency of $\Omega_{\text {Sync }}$. Each colored curve corresponds to a different value of the initial delay $(\Delta t)$. The magnitude of the vertical axis has been chosen to be $\sin \left(\Omega_{\text {Sync }}\right)$ to illustrate that the trajectory in the phase space is a closed cycle.

found in the same position. In that case the phases of the two oscillators are the same for every sampled point of the traces, which is consistent with the experimental observations. Each of the colored curves in Fig. 3(c) corresponds to a stroboscopic sampling at $\Omega_{\text {Sync }}$ for a specific value of the initial delay $[\Delta t$; see Fig. 3(b)]. The points remain in a confined volume of the phase space that is dominated by the experimental noise. By changing $\Delta t$ from 0 to $2 \pi / \Omega_{\text {Sync }}$ it is possible to rebuild the limit cycle of the synchronized state in the optical transmission phase space. An important point to consider here is that the maximum mechanical deformation of an OMC is achieved in between the two minima of the optical transmission, as is shown in Fig. 3(b) (see also Supplemental Material [32]). We can thus conclude that the temporal delay between the mechanical signals of each oscillator is about half of the period, i.e., there is a $\pi$ phase shift between the mechanical oscillations.

Finally, we explore the effect of illuminating the master cavity region with a top heating laser. Our previous work on a single OMC showed that its dynamical state is modified when the laser is switched on because of photothermal effects [41]. Now, before switching on the top pumping, we set the parameters of the laser driving the slave in a way that its dynamical state is a mechanical lasing regime at $\Omega_{S}$ activated by the 3rd harmonic of the optical force [34]. The master is then driven to a mechanical lasing regime using

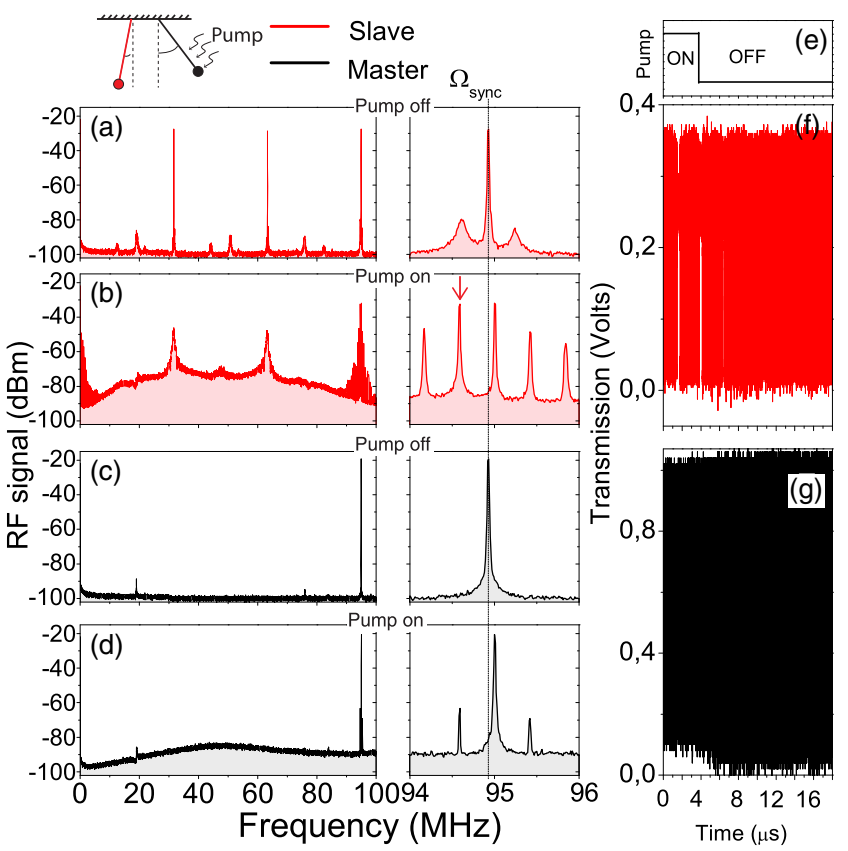

FIG. 4. Switching on and off the synchronized state. (a)(d) Radio-frequency spectra of the transmitted signal of the slave [(a) and (b)] and master [(c) and (d)] when the pump is off [(a) and (c)] and on [(b) and (d)]. The right panels correspond to an enlargement in the spectral region around the mechanical modes frequencies. The synchronized state frequency $\left(\Omega_{\text {Sync }}\right)$ is highlighted with a vertical line on the right panels. A vertical red arrow indicates $\Omega_{S}$ in the right panel (b). (e) Temporal trace of the top pumping laser when the modulation is active. (f)-(g) Temporal traces of the optical transmission of the master and slave cavities [(f) and (g), respectively] when the master is illuminated with a modulated top pumping laser (see also Supplemental Material [32]).

the 1st harmonic of the force, where both cavities synchronize their mechanical oscillations at $\Omega_{\text {Sync }}$ [Figs. 4(a) and 4(c)]. It is important to note that, under this configuration, the first harmonic of the optical transmission of the slave is at $\Omega_{\text {Sync }} / 3$ and that there are no signs of the 1 st and 2nd harmonic of that signal when measuring the master. The latter observation is a conclusive evidence of a pure mechanical coupling between the OMCs, the leaked mechanical energy being enough to be transduced despite the rather low cross coupling $g_{o}$ (see Supplemental Material [32]).

When the heating laser is switched on the synchronized state is spoiled and a dynamical state similar to the one reported in Figs. 2(c) and 2(d) is achieved in the slave [Fig. 4(b)]. The master still shows a coherent tone at $\Omega_{M}$ [Fig. 4(d)] but narrow symmetric sidebands appear associated with frequency beating with the coherent mechanical oscillation of the slave. Although the elastic constants of the master are slightly relaxed due to heating, $\Omega_{M}$ shifts to a higher frequency value. This counterintuitive effect is a result of the attenuation of the frequency pulling effect 
induced by the coupling to the slave mechanical dynamics; i.e., when the synchronized state is spoiled the coupling is reduced and $\Omega_{M}$ goes towards the eigenfrequency of the master at that temperature. The same phenomenon occurs to $\Omega_{S}$ in the opposite direction and in a much larger scale [red arrow of Fig. 4(b)]. By activating the modulation of the top pumping laser following the temporal profile of Fig. 4(e) it is possible to dynamically switch between the two states described above. The temporal traces when collecting the slave and master optical signals are reported in Figs. 4(f) and 4(g), respectively. When the pump is switched off the OMCs take several microseconds to adjust their oscillation rhythms and stabilize the mechanical synchronized state, which is a direct consequence of their weak interaction.

In conclusion, we have unequivocally demonstrated synchronization of the mechanical oscillations of a pair of optomechanical crystal cavities by introducing a weak mechanical coupling. Provided that our numerical model indicates that the observed features are compatible with a reactive type of coupling (see Supplemental Material [32]), further experimental studies will unveil whether the transition to synchronization is through phase locking or suppression of the natural dynamics, which are the two mechanisms of synchronization in reactively coupled oscillators [3]. Finally, we have demonstrated that the synchronization dynamics can be dynamically switched off and on by introducing an external heating source on the master cavity.

The system presented here could be upscaled to realize complex networks comprising many more nodes without substantially increasing the technological requirements. For instance, one could easily think of a silicon chip integrating an array of optomechanical cavities accessed optically via integrated waveguides [42,43] and interconnected via mechanical links. Therefore, our results are the first step towards building networks of coupled optomechanical crystal cavities able to display collective dynamics prone to be modified by addressing single structures with external perturbations. These rather unique features are to be exploited in neuromorphic photonic computing applications [44], for instance, for pattern recognition tasks or more complex cognitive processing. Eventually it will be possible to experimentally investigate the limits, in terms of network complexity, of coherent collective behaviors and the intriguing transition towards incoherence, where the socalled Chimera states $[45,46]$ of synchronous and incoherent behavior are expected to emerge.

This work was supported by the European Commission project PHENOMEN (H2020-EU-713450), the Spanish Severo Ochoa Excellence program, and the MINECO project PHENTOM (FIS2015-70862-P). D. N. U., G. A., and M. F.C. gratefully acknowledge the support of a Ramón y Cajal postdoctoral fellowship (RYC-201415392), a BIST studentship, and a Severo Ochoa studentship, respectively. The authors thank Dr. G. Whitworth for his careful and critical reading of the manuscript.

*These authors contributed equally to this work. †navarro@ub.edu

[1] C. Huygens, Horologium Oscillatorium (The Pendulum Clock) (Apud F. Muguet, Paris, 1673); R. J. Blackwell, Transl. (Iowa State University Press, Ames, IA, 1986)].

[2] A. Pikovsky, M. Rosenblum, and J. Kurths, Synchronization: A Universal Concept in Nonlinear Sciences (Cambridge University Press, Cambridge, England, 2003).

[3] A. Balanov, N. Janson, and D. Postnov, Synchronization: From Simple to Complex (Springer, New York, 2009).

[4] M. Fussenegger, Synthetic biology: Synchronized bacterial clocks, Nature (London) 463, 301 (2010).

[5] F. K. Lamb, J. J. Aly, M. Cook, and D. Q. Lamb, Synchronization of magnetic stars in binary systems, Astrophys. J. 274, L71 (1983).

[6] S.H. Strogatz, Exploring complex networks, Nature (London) 410, 268 (2001).

[7] F. Dörfler and F. Bullo, Synchronization in complex networks of phase oscillators: A survey, Automatica 50, 1539 (2014).

[8] L. M. Pecora, F. Sorrentino, A. M. Hagerstrom, T. E. Murphy, and R. Roy, Cluster synchronization and isolated desynchronization in complex networks with symmetries, Nat. Commun. 5, 4079 (2014).

[9] F. C. Hoppensteadt and E. Izhikevich, Oscillatory Neurocomputers with Dynamic Connectivity, Phys. Rev. Lett. 82, 2983 (1999).

[10] S. B. Shim, M. Imboden, and P. Mohanty, Synchronized oscillation in coupled nanomechanical oscillators, Science 316, 95 (2007).

[11] D. K. Agrawal, J. Woodhouse, and A. A. Seshia, Observation of Locked Phase Dynamics and Enhanced Frequency Stability in Synchronized Micromechanical Oscillators, Phys. Rev. Lett. 111, 084101 (2013).

[12] M. H. Matheny, M. Grau, L. G. Villanueva, R. B. Karabalin, M. C Cross, and M. L. Roukes, Phase Synchronization of Two Anharmonic Nanomechanical Oscillators, Phys. Rev. Lett. 112, 014101 (2014).

[13] A. S. Tanembaum and M. Van Steen, Distributed Systems: Principles and Paradigms (Prentice Hall, NJ, 2002).

[14] M. S. Hanay, S. Kelber, A. K. Naik, D. Chi, S. Hentz, E. C. Bullard, E. Colinet, L. Duraffourg, and L. M. Roukes, Single-protein nanomechanical mass spectrometry in real time, Nat. Nanotechnol. 7, 602 (2012).

[15] I. Bargantin, E. B. Myers, J. S. Aldridge, C. Marcoux, P. Brianceau, L. Duraffourg, E. Colinet, S. Hentz, P. Andreucci, and M. L. Roukes, Large-scale integration of nanoelectromechanical systems for gas sensing applications, Nano Lett. 12, 1269 (2012).

[16] M. Li, E. B. Myers, H. X. Tang, S. J. Aldridge, H. C. McCaig, J. J. Whiting, R. J. Simonson, N. S. Lewis, and M. L. Roukes, Nanoelectromechanical resonator arrays for 
ultrafast, gas-phase chromatographic chemical analysis, Nano Lett. 10, 3899 (2010).

[17] D. Rugar, R. Budakian, H. J. Mamin, and B. W. Chui, Single spin detection by magnetic resonance force microscopy, Nature (London) 430, 329 (2004).

[18] M. Aspelmeyer, T. J. Kippenberg, and F. Marquardt, Cavity optomechanics, Rev. Mod. Phys. 86, 1391 (2014).

[19] A. Safavi-Naeini, S. Grblacher, J. T. Hill, J. Chan, M. Aspelmeyer, and O. Painter, Squeezed light from a silicon micromechanical resonator, Nature (London) 500, 185 (2013).

[20] R. Riedinger, S. Hong, R. A. Norte, J. A. Slater, J. Shang, A. G. Krause, V. Anant, M. Aspelmayer, and S. Grblacher, Non-classical correlations between single photons and phonons from a mechanical oscillator, Nature (London) 530, 313 (2016).

[21] K. C. Balram, M. I. Davano, J. D. Song, and K. Srinivasan, Coherent coupling between radiofrequency, optical and acoustic waves in piezo-optomechanical circuits, Nat. Photonics 10, 346 (2016)

[22] J. D. Teufel, T. Donner, D. Li, J. W. Harlow, M. S. Allram, K. Cicak, A. J. Sirois, J. D. Whittaker, W. Lehnert, and R.W. Simmonds, Sideband cooling of micromechanical motion to the quantum ground state, Nature (London) $\mathbf{4 7 5}$, 359 (2011).

[23] G. Heinrich, M. Ludwig, J. Qian, B. Kubala, and F. Marquardt, Collective Dynamics in Optomechanical Arrays, Phys. Rev. Lett. 107, 043603 (2011).

[24] V. Peano, C. Brendel, M. Schmidt, and F. Marquardt, Topological Phases of Sound and Light, Phys. Rev. X 5, 031011 (2015).

[25] M. Zhang, G. S. Wiederhecker, S. Manipatruni, A. Barnard, P. McEuen, and M. Lipson, Synchronization of Micromechanical Oscillators using Light, Phys. Rev. Lett. 109, 233906 (2012).

[26] M. Zhang, S. Shah, J. Cardenas, and M. Lipson, Synchronization and Phase Noise Reduction in Micromechanical Oscillator Arrays Coupled through Light, Phys. Rev. Lett. 115, 163902 (2015).

[27] M. Bagheri, M. Poot, L. Fan, F. Marquardt, and H. X. Tang, Photonic Cavity Synchronization of Nanomechanical Oscillators, Phys. Rev. Lett. 111, 213902 (2013).

[28] E. Gil-Santos, M. Labousse, C. Baker, A. Goetschy, W. Hease, C. Gomez, A. Lemaitre, C. Ciuti, and I. Favero, Light-Mediated Cascaded Locking of Multiple NanoOptomechanical Oscillators, Phys. Rev. Lett. 118, 063605 (2017).

[29] S. Shah, M. Zhang, R. Rand, and M. Lipson, Master-Slave Locking of Optomechanical Oscillators Over a Long Distance, Phys. Rev. Lett. 114, 113602 (2015).

[30] S. Shah, M. Zhang, R. Rand, and M. Lipson, Long-range synchronization of nanomechanical oscillators with light, arXiv:1511.08536.

[31] J. K. Jang, A. Klenner, X. Ji, Y. Okawachi, M. Lipson, and A. L. Gaeta, Synchronization of coupled optical microresonators, Nat. Photonics 12, 688693 (2018).
[32] See Supplemental Material at http://link.aps.org/ supplemental/10.1103/PhysRevLett.123.017402 for details on the fabrication process, finite element method simulations, numerical model describing the dynamics of the coupled system and temporal traces of the switching experiment.

[33] T. J. Johnson, M. Borselli, and O. Painter, Self-induced optical modulation of the transmission through a high-Q silicon microdisk resonator, Opt. Express 14, 817 (2006).

[34] D. Navarro-Urrios, N. E. Capuj, J. Gomis-Bresco, F. Alzina, A. Pitanti, A. Griol, A. Martinez, and C. M. Sotomayor Torres, A self-stabilized coherent phonon source driven by optical forces, Sci. Rep. 5, 15733 (2015).

[35] D. Navarro-Urrios, N. E. Capuj, M. F. Colombano, P. D. Garcia, M. Sledzinska, F. Alzina, A. Griol, A. Martinez, and C. M. Sotomayor Torres, Nonlinear dynamics and chaos in an optomechanical beam, Nat. Commun. 8, 14965 (2017).

[36] S. G. Johnson, M. Ibanescu, M. Skorobogatiy, O. Wiesberg, J. D. Joannopoulos, and Y. Fink, Perturbation theory for Maxwells equations with shifting material boundaries, Phys. Rev. E 65, 066611 (2002).

[37] J. Chan, A. Safavi-Naeini, J. T. Hill, S. Meenehan, and O. Painter, Optimized optomechanical crystal cavity with acoustic radiation shield, Appl. Phys. Lett. 101, 081115 (2012).

[38] Y. Pennec, V. Laude, N. Papanikolaou, B. Djafari-Rouhani, M. Oudich, S. El Jallal, J. C. Beugnot, J. M. Escalante, and A. Marinez, Modeling light-sound interaction in nanoscale cavities and waveguides, Nanophotonics 3, 413 (2014).

[39] A. Balanov, N. Janson, and D. Postnov, Synchronization: From simple to complex (Springer, New York, 2009), p. 91.

[40] R. Leijssen, G. La Gala, L. Freisem, J. Muhonen, and E. Verhagen, Nonlinear cavity optomechanics with nanomechanical thermal fluctuations, Nat. Commun. 8, 16024 (2017).

[41] J. Maire, G. Arregui, N. E. Capuj, M. F. Colombano, A. Griol, A. Martinez, C. M. Sotomayor-Torres, and D. Navarro-Urrios, Optical modulation of coherent phonon emission in optomechanical cavities, APL Photonics 3, 126102 (2018).

[42] K. Fang, M. H. Matheny, X. Luan, and O. Painter, Optical transduction and routing of microwave phonons in cavity-optomechanical circuits, Nat. Photonics 10, 489 (2016).

[43] J. Bochmann, A. Vainsencher, D. D. Awschalom, and A. N. Cleland, Nanomechanical coupling between microwave and optical photons, Nat. Phys. 9, 712 (2013).

[44] Y. Shen, N. C. Harris, S. Skirlo, M. Prabhu, T. Baehr-Jones, M. Hochberg, X. Sun, S. Zhao, H. Larochelle, D. Englund, and M. Soljai, Deep learning with coherent nanophotonic circuits, Nat. Photonics 11, 441 (2017).

[45] D. J. Watts and S. H. Strogatz, Collective dynamics of small-world networks, Nature (London) 393, 440 (1998).

[46] M. J. Pannaggio and D. M. Abrams, Chimera states: Coexistence of coherence and incoherence in networks of coupled oscillators, Nonlinearity 28, R67 (2015). 